\title{
Serovar distribution, antimicrobial resistance profiles, and PFGE typing of Salmonella enterica strains isolated from 2007-2012 in Guangdong, China
}

Bixia Ke ${ }^{1+}$, Jiufeng Sun ${ }^{1,2+}$, Dongmei He${ }^{1}$, Xiaocui $\mathrm{Li}^{1}$, Zhaoming Liang ${ }^{1}$ and Chang-wen Ke ${ }^{1 *}$

\begin{abstract}
Background: Salmonella enterica includes the major serovars associated with human salmonellosis. In this study, 1764 clinical Salmonella enterica isolates from diarrhea outpatients were collected from fifteen cities in Guangdong province, China, between 2007 and 2012. These isolates represent all of the Salmonella isolates collected from the province during that period.
\end{abstract}

Methods: The isolates were characterized by serovar determination, antimicrobial susceptibility tests and PFGE fingerprint typing.

Results: The serovar distribution results demonstrated that Salmonella Typhimurium $(n=523,29.65 \%)$ and Salmonella $4,5,12: i:-(n=244,13.83 \%)$ are the most common serovars causing infant salmonellosis, whereas Salmonella Enteritidis $(n=257,14.57 \%$ ) mainly causes human salmonellosis in adults. The serovar shift from Salmonella Enteritidis to Salmonella Typhimurium occurred in 2008. Antimicrobial susceptibility data showed a high burden of multidrug resistance (MDR) $(n=1128,56.58 \%)$, and a 20\%-30\% increase in the number of isolates resistant to ciprofloxacin $(n=142,8.05 \%)$ and third-generation cephalosporins ( $n=88,4.99 \%)$ from 2007-2012. Only $9.97 \%$ of isolates $(n=176)$ were fully susceptible to all agents tested. A high burden of MDR was observed in Salmonella Typhimurium and Salmonella 4,5,12:i:- for all age groups, and a reduced susceptibility to third-generation cephalosporins and quinolones occurred particularly in infants ( $\leq 6$ years). The dominant PFGE patterns were JPXX01.GD0004, JEGX01.GD0006-7 and JNGX01.GD0006-7. ACSSUT was the predominant MDR profile in the Salmonella Typhimurium \& 4,5,12:i:- complexes, while ASSuT-Nal and ASSu-Nal were the major MDR profiles in Salmonella Enteritidis. The predominant PFGE patterns of the Salmonella Typhimurium \& 4,5,12:i:- complexes and Salmonella Stanley were most prevalent in infants ( $\leq 6$ years). However, no obvious relationship was observed between these PFGE profiles and geographic location.

Conclusions: These data reveal the serovar distribution of isolates recovered from diarrhea patients, the characteristics of resistant strains and fingerprint typing in Guangdong from 2007 to 2012. These results highlight a serovar shift and a worrying percentage of MDR strains with increasing resistance to quinolones and third-generation cephalosporins. Thus, continued surveillance of Salmonella and their MDR profiles using combined molecular tools and efforts to control the rapid increase in antimicrobial resistance among Salmonella in Guangdong are needed.

Keywords: Salmonella enterica, Non-typhoidal Salmonella, Antimicrobial susceptibility, Ciprofloxacin, Cephalosporins, MDR, PFGE

\footnotetext{
* Correspondence: kecw2011@sina.com

'Equal contributors

Institute of Microbiology, Guangdong Provincial Center for Disease Control and Prevention, 511430 Guangzhou, China

Full list of author information is available at the end of the article
}

\section{Biomed Central}

(c) 2014 Ke et al.; licensee BioMed Central Ltd. This is an Open Access article distributed under the terms of the Creative Commons Attribution License (http://creativecommons.org/licenses/by/4.0), which permits unrestricted use, distribution, and reproduction in any medium, provided the original work is properly credited. The Creative Commons Public Domain Dedication waiver (http://creativecommons.org/publicdomain/zero/1.0/) applies to the data made available in this article, unless otherwise stated. 


\section{Background}

The genus Salmonella currently includes two species, Salmonella enterica and Salmonella bongori. Salmonella enterica is further divided into subspecies I (enterica), II (salamae), IIIa (arizonae), IIIb (diarizonae), IV (houtenae), and VI (indica). Serotyping is the traditional method for the subtyping and the differentiation of Salmonella isolates based on the Kauffmann-White scheme. Over 2500 Salmonella serotypes are currently known [1]. Salmonella enterica, the etiological agent of salmonellosis, is recognized as an endemic food-borne pathogen that causes a heavy global socioeconomic burden [2-4]. Salmonella enterica infections result in diverse clinical manifestations. Typhoid fever, caused by Salmonella Typhi and Salmonella Paratyphi, is a bacteremic illness which occurs in strong, healthy hosts in addition to immunocompromised hosts and infants and is essentially dependent on the bacterial inoculum/infectious dose [5]. Non-typhoidal Salmonella (NTS) serovars (e.g., Salmonella Enteritidis, Salmonella Typhimurium and Salmonella 4,5,12:i:-) normally cause self-limiting diarrhea or gastroenteritis, which results in a significant disease burden, with an estimated 2.8 million cases of diarrhea and 93.8 million cases of gastroenteritis, worldwide, and 155,000 deaths each year [6]. The host range of NTS serovars is broad, including poultry and cattle. Infection caused by NTS is commonly due to contamination in the food chain and food poisoning in developing countries. Occasionally, NTS serovars cause secondary bacteremia, while in immunocompromised patients in sub-Saharan Africa, they cause high rates of bacteremia in children below 5 years old as well as those with HIV infection [5,6].

Typhoid Salmonella caused 216,000 deaths globally in 2010 [2], whereas most cases of invasive, non-typhoid salmonellosis occur occasionally and are due to either Salmonella Typhimurium or Salmonella Enteritidis [7]. The resistance of NTS to ampicillin, chloramphenicol and sulphamethoxazole is usually high in Africa and the USA $[8,9]$. The treatment of NTS, therefore, increasingly relies on fluoroquinolones or third-generation cephalosporins; however, these treatment options are threatened by reduced bacterial susceptibility to fluoroquinolones and extended-spectrum beta-lactam antibiotics, respectively $[9,10]$. In Hong Kong, China, the rate of infections by NTS has been increasing since 1989, and the most common Salmonella serovar is Enteritidis [11]. The antimicrobial susceptibilities of 275 Salmonella Enteritidis strains isolated from 1986 to 1996 show that over 99\% of the isolates are susceptible to 17 of the 19 antimicrobial agents tested [12]. From 2005 to 2010, Salmonella Enteritidis was the predominant serovar in Hong Kong; in that time, the strains that were not susceptible to ciprofloxacin increased significantly, from $39.3 \%$ to $63 \%$, and the percentage of multidrug resistant (MDR) strains increased from $17.8 \%$ to $36.2 \%$ [13]. In an inland province, Henan, the two most common serovars among the isolates collected during 2006 and 2007 were Salmonella Typhimurium (27\%) and Salmonella Enteritidis (17\%) [14]. Our previous study on Salmonella infection in diarrhea patients in Guangdong province showed that Salmonella Typhimurium and Enteritidis were the most common serovars. Eighty percent of the 229 isolates were susceptible to cephalosporins and quinolones, while $59.3 \%$ of them were multidrug resistant strains [15-18]. However, these studies did not provide sufficient information concerning prevalence, serovar distribution, and antimicrobial susceptibility in Guangdong province. Reduced quinolone- and third-generation cephalosporin- susceptibility in NTS is also poorly documented [10,19].

The current study describes serovar profiles and potential profile shifts, characterizes MDR isolates with a special focus on susceptibility to quinolones and third-generation cephalosporins and reveals associations between MDR profiles and PFGE fingerprint pattern.

\section{Methods}

\section{Ethics statement}

Ethical approval was granted by the Ethical Committee of the Guangdong Provincial Center for Disease Control and Prevention. The present study complies with the World Health Organization and international guidelines on global surveillance [20]. The data have been reviewed and analyzed anonymously.

\section{Sampling, bacterial culture and identification}

According to the Global Salmonella Surveillance program, all the cities in Guangdong Province were included in this system (Figure 1), while 15 of 21 cities submitted the samples to our center for Salmonella surveillance. The recruitment of patients followed specific guidelines. Patients who had two of the following three symptoms were chosen for the study: 1 ) diarrhea more than three times within 24 hours, with watery stools; 2) fever $>38^{\circ} \mathrm{C}$, headache, chills, malaise; 3) diarrhea with vomiting, abdominal pain, and watery stools. Stool samples were collected from clinical diarrhea outpatients of hospitals in fifteen cities from 2007 to 2012 (Figure 1). All of the samples $(42,889)$ were cultured in local hospitals using solid broth agar media (Huankai, Guangzhou, China) for the initial evaluation of Salmonella growth, and then suspicious colonies were picked into vials containing semi-solid Rappaport Vassiliadis Medium (OXOID, France), and shipped to Guangdong Provincial Center for Disease Control and Prevention (Guangzhou, China) at room temperature, where they were incubated daily and checked for growth. The cultures that grew were Gram stained, subcultured and identified at the species level by standard biochemical methods, including the characteristic growth on Kligler Iron Agar (Huankai, Guangzhou, China), tests for urease, oxidase, b- 


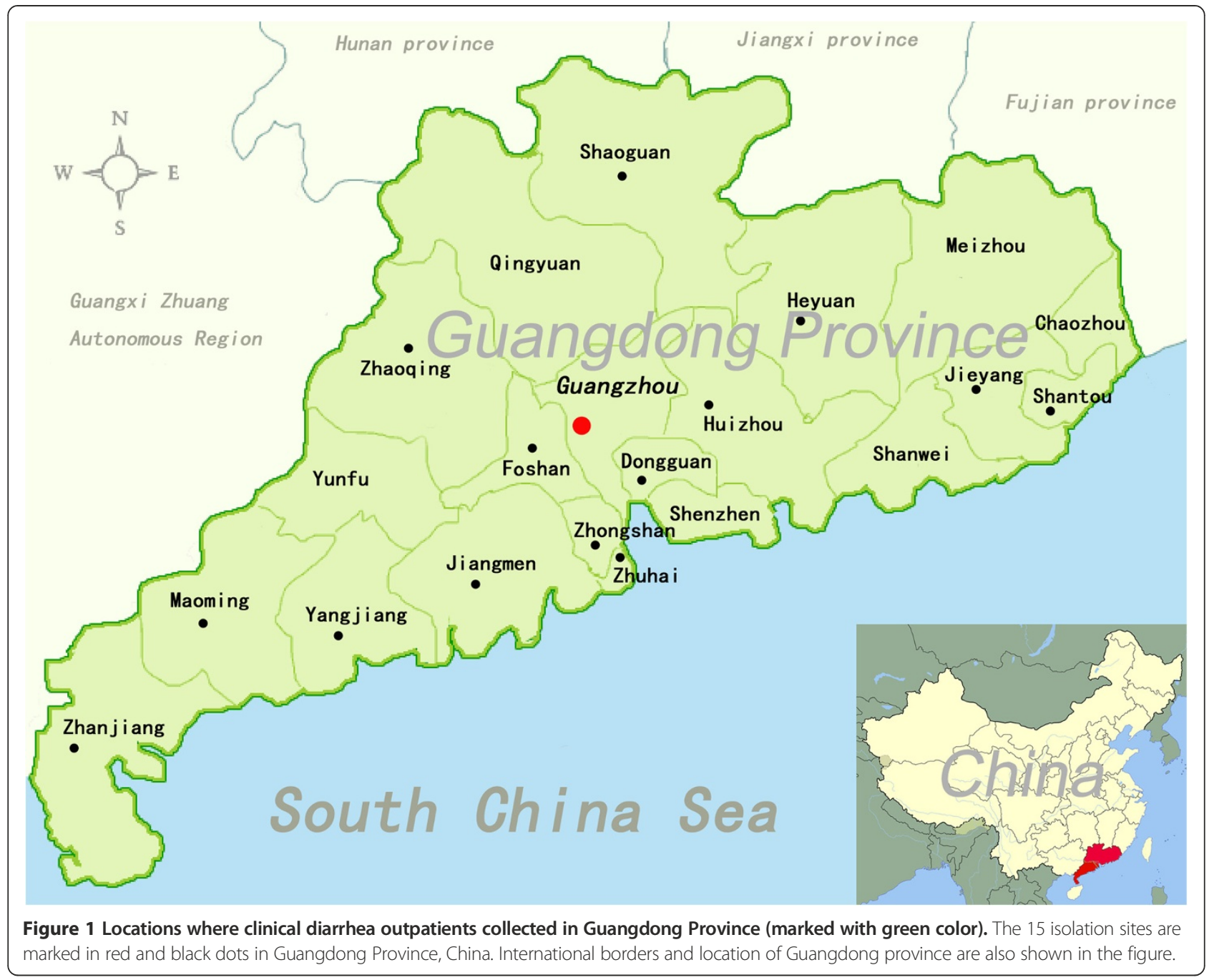

galactosidase and indole production, and positive tests for lysine decarboxylase (the technician training course was first launched on Apr, 2007, and updated on Sep, 2009). In total, 1764 stool samples showed positive results for Salmonella growth. The serovar of the Salmonella isolates was confirmed by slide agglutination with commercial monospecific anti-sera (Sifin, Berlin, Germany), following the White-Kauffmann-Le Minor scheme [21]. The outbreak isolates were collected separately, and all the strains collected as described above were considered epidemiologically unrelated. Age, gender and geographic origin were recorded as part of the standard information present on the laboratory request forms.

\section{Antimicrobial susceptibility}

The antimicrobial susceptibility test was performed according to the guidelines of the Clinical and Laboratory Standards Institute (CLSI) [22]. The agar diffusion assays were performed on Muller-Hinton agar with disks containing seven classes of antimicrobial agents (aminoglycoside, cephalosporin, penicillin, quinolone, tetracycline, amphenicol, and sulfonamide). The specific antimicrobials used were: streptomycin $10 \mu \mathrm{g}(\mathrm{S})$, gentamicin $10 \mu \mathrm{g}(\mathrm{G})$, ceftazidime $30 \mu \mathrm{g}(\mathrm{Caz})$, cefotaxime $30 \mu \mathrm{g}(\mathrm{Ctx})$, cefepime $30 \mu \mathrm{g}$ (Fep), ampicillin $10 \mu \mathrm{g}(\mathrm{A})$, nalidixic acid $30 \mu \mathrm{g}(\mathrm{Nal})$, ciprofloxacin $5 \mu \mathrm{g}(\mathrm{Cp})$, tetracycline $30 \mu \mathrm{g}(\mathrm{T})$, chloramphenicol $30 \mu \mathrm{g}(\mathrm{C})$, sulfamethoxazole $300 \mu \mathrm{g}(\mathrm{Su})$, and trimethoprim $5 \mu \mathrm{g}(\mathrm{Tm})$. The results were interpreted as resistant, intermediate or susceptible, according to the CLSI guidelines [22]. Escherichia coli ATCC25922 was used as quality control organism. MDR was identified as resistance to more than three classes of antimicrobials. The antimicrobial resistance pattern and the sources of the strains are shown in Additional file 1: Table S1.

\section{Pulsed field gel electrophoresis}

The pulsed field gel electrophoresis (PFGE) results were taken from the routine surveillance data in our lab. PFGE was performed according to the PulseNet protocol for Salmonella [23], using XbaI as restriction 
enzyme (New England Biolabs, Leusden, The Netherlands). The cluster analysis was performed with Bionumerics 5.1 (Applied Maths NV, Sint-Martens-Latem, Belgium) using the Dice similarity coefficient and UPMGA (unweighted pair group method using average linkages) dendrogram type (optimization $0.50 \%$, position tolerance $1.50 \%$ ).

\section{Data analysis}

All data were entered in an Excel database (Microsoft Corporation, Redmond, Washington, USA). Proportions were assessed for statistical significance using the Chi square test or Fisher's exact test, considering $p<0.05$ as significant. If the data were normally distributed, the mean values of two groups were compared with Student's t-test; otherwise, median values were compared with the Mann-Whitney rank sum test (Stata 10, Stat Corp, Texas, USA).

\section{Results}

Serovars and epidemiological data

In total, 1,764 Salmonella isolates were successfully isolated from 42,889 stool samples, which were collected from diarrhea outpatients in 15 cities in Guangdong Province from 2007-2012 (Additional file 1: Table S1). The serovar identification showed that these isolates could be classified into 128 serovars. The most prevalent strains isolated during the test period were NTS (Table 1). The predominant NTS consisted of Salmonella Typhimurium ( $\mathrm{n}=523,29.65 \%)$, Salmonella Enteritidis ( $\mathrm{n}=257,14.57 \%)$, Salmonella 4,5,12: i:- $(\mathrm{n}=244,13.83 \%)$, Salmonella Stanley $(\mathrm{n}=167,9.47 \%)$, Salmonella Derby $(\mathrm{n}=46,2.61 \%)$, Salmonella Rissen ( $\mathrm{n}=$ 36, 2.04\%), Salmonella Weltevreden ( $\mathrm{n}=35,1.98 \%)$, Salmonella Infantis $(\mathrm{n}=28,1.58 \%)$, Salmonella Agona $(\mathrm{n}=24$,
1.36\%), Salmonella Albany ( $\mathrm{n}=22,1.25 \%)$ and Salmonella Newport ( $\mathrm{n}=19,1.08 \%$ ) (Table 1). The incidence of Salmonella infections caused by the top 11 NTS serovars ( $\mathrm{n}=$ 1401, 79.42\%) showed an increasing trend from 2007 to 2012, except for Salmonella Typhimurium in 2010 (Table 1). In 2007, Salmonella Enteritidis was the major causative serovar in diarrhea outpatients $(\mathrm{n}=14,26.4 \%)$, while in 2008, Salmonella Typhimurium $(\mathrm{n}=20,29.85 \%)$ became more frequently isolated than Salmonella Enteritidis $(n=17$, 25.30\%). From that point, Salmonella Typhimurium and Salmonella 4,5,12:i:- were the predominant endemic serovars in diarrhea outpatients in Guangdong (Figure 2).

The male/female ratio of the patients was 1.37 (1020 male, 744 female). The age distribution of the patients with the predominant Salmonella serovars is illustrated in Figure 3. The median patient age was 0.92 years (interquartile range: $0.58-4$ years). The age-associated stratified analysis showed that Salmonella Typhimurium and Salmonella 4,5,12:i:were the predominant infectious agents in infants ( $<6$ years), whereas Salmonella Enteritidis was the predominant serovar in adults (>15 years). However, there was no statistically significant difference between the median age of Salmonella Typhimurium, Salmonella 4,5,12:i:- and Salmonella Enteritidis patients ( $p>0.05$, Mann-Whitney Rank Sum Test).

\section{Antimicrobial susceptibility}

The antimicrobial resistance profiles are shown in Additional file 1: Table S1. Of all the isolates, $63.49 \%$, $59.97 \%, 59.64 \%, 58.78 \%$ and $49.77 \%(\mathrm{n}=1120,1058$, 1052, 1037 and 878) of the isolates were resistant to sulfamethoxazole, tetracycline, nalidixic acid, ampicillin and streptomycin, respectively (Table 2). Four of the

Table 1 Distribution of the predominant serovars of 1764 Salmonella enterica isolates from humans with infections in Guangdong Province, China from 2007 to 2012

\begin{tabular}{|c|c|c|c|c|c|c|c|c|}
\hline Salmonella enterica serovars & 2007 & 2008 & 2009 & 2010 & 2011 & 2012 & In total & $\%$ of total isolates \\
\hline Enteritidis & 14 & 17 & 28 & 31 & 48 & 119 & 257 & 14.57 \\
\hline Typhimurium & 8 & 20 & 40 & 129 & 110 & 216 & 523 & 29.65 \\
\hline 1,4,5,12:i:- & 0 & 6 & 4 & 16 & 79 & 139 & 244 & 13.83 \\
\hline Stanley & 3 & 5 & 14 & 14 & 38 & 93 & 167 & 9.47 \\
\hline Derby & 2 & 3 & 2 & 7 & 9 & 23 & 46 & 2.61 \\
\hline Rissen & 0 & 0 & 0 & 0 & 12 & 24 & 36 & 2.04 \\
\hline Weltevreden & 0 & 0 & 2 & 9 & 7 & 17 & 35 & 1.98 \\
\hline Infantis & 0 & 0 & 3 & 4 & 12 & 9 & 28 & 1.58 \\
\hline Agona & 1 & 2 & 4 & 0 & 3 & 14 & 24 & 1.36 \\
\hline Albany & 0 & 1 & 1 & 5 & 6 & 9 & 22 & 1.25 \\
\hline Newport & 1 & 0 & 3 & 6 & 0 & 9 & 19 & 1.08 \\
\hline Typhi & 0 & 0 & 1 & 1 & 1 & 1 & 4 & 0.23 \\
\hline Paratyphi $(A+B+C)$ & 1 (B) & 0 & $3(\mathrm{~B})$ & $5(2 A+2 B+1 C)$ & $3(\mathrm{~B})$ & $12(3 A+9 B)$ & 24 & 1.36 \\
\hline other 114 NTS serovars & 23 & 14 & 26 & 54 & 61 & 158 & 335 & 18.99 \\
\hline Total & 53 & 68 & 131 & 281 & 389 & 843 & 1764 & 100 \\
\hline
\end{tabular}




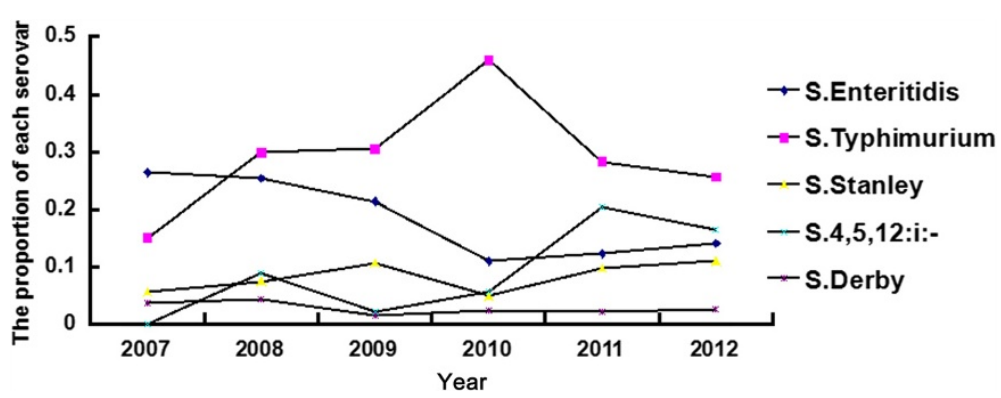

Figure 2 The proportion of top 5 serovars among the total isolates from 2007 to 2012. Each data point means the proportion of each serovar in the present year (indicated as percentage in Y-axis), different serovars are indicated using different symbols as illustrated in figure.

twelve antimicrobial agents, ceftazidime, cefotaxime, cefepime and ciprofloxacin, showed high susceptibility (>90\%), while the trend for increased resistance was much greater for these four agents than for the other eight drugs (20-30\%) (Figure 4A, red axis). Within typhoid Salmonella, four of twenty-eight isolates were MDR strains, while only ESS12371 and ESS12984 were resistant to ciprofloxacin and cephalosporins (ceftazidime, cefotaxime and cefepime), respectively (Additional file 1: Table S1). In the NTS isolates, the proportion of Salmonella Typhimurium and Salmonella 4,5,12:i:- resistant to trimethoprim, tetracycline, gentamicin, chloramphenicol, cefotaxime and ciprofloxacin were significantly higher $(p<0.05)$ than Salmonella Enteritidis (Table 2). However, $8.05 \%$ of all isolates $(\mathrm{n}=1764)$, showed a reduced susceptibility to ciprofloxacin (Salmonella Typhimurium $[\mathrm{n}=60]$, Salmonella 4,5,12:i:- $[\mathrm{n}=28]$, Salmonella Enteritidis [n = 11], Salmonella Stanley $[\mathrm{n}=8]$ and other 21 serovars [n =35]) (Table 2), whereas $4.99 \%(88 / 1764)$ of them were resistant to ceftazidime, cefotaxime and cefepime at same time. Resistance to cefepime alone occurred in $9.58 \%$ of the isolates $(169 / 1764$, Salmonella Typhimurium [ $\mathrm{n}=69$ ], Salmonella 4,5,12:i:- [n = 29], Salmonella Enteritidis [n =18], Salmonella Stanley [n =9] and other 24 serovars, $[\mathrm{n}=44])($ Table 2$)$.
MDR occurred in $56.51 \%$ (997/1764) of all isolates. The reduced susceptibility was observed in all tested antimicrobial agents from 2007 to 2012 with few exceptions (Salmonella Enteritidis from 2008 to 2010 and Salmonella Stanley from 2007 to 2008) (Figure 4B). The proportion of MDR isolates increased yearly in Salmonella Typhimurium and Salmonella 4,5,12:i:- (>80\%), while this proportion declined in Salmonella Enteritidis. The ACSSuT is the most prevalent MDR pattern (27.04\%, 477/1764), particularly in Salmonella Typhimurium and Salmonella 4,5,12:i- (74.42\%, 291/391). Two Salmonella Typhimurium and one Salmonella 4,5,12:i:- isolate(s) resistant to all twelve antimicrobial agents were obtained (marked red in Additional file 1: Table S1). Only 9.97\% (176/1764) of isolates were fully susceptible to all tested agents (Table 2). The ratio of MDR between the sampling locations was comparable (46.5-71.4\%) (Table 2). No obvious relationships between MDR or resistance to single antimicrobial agents and sampling area were observed within these isolates.

The age-associated stratified analysis showed severe MDR in Salmonella Typhimurium and Salmonella 4,5,12: i:- for all age groups, which was even worse in infants. Reduced susceptibility occurred in infants for the tested thirdgeneration cephalosporin and quinolone (Figure 4C).

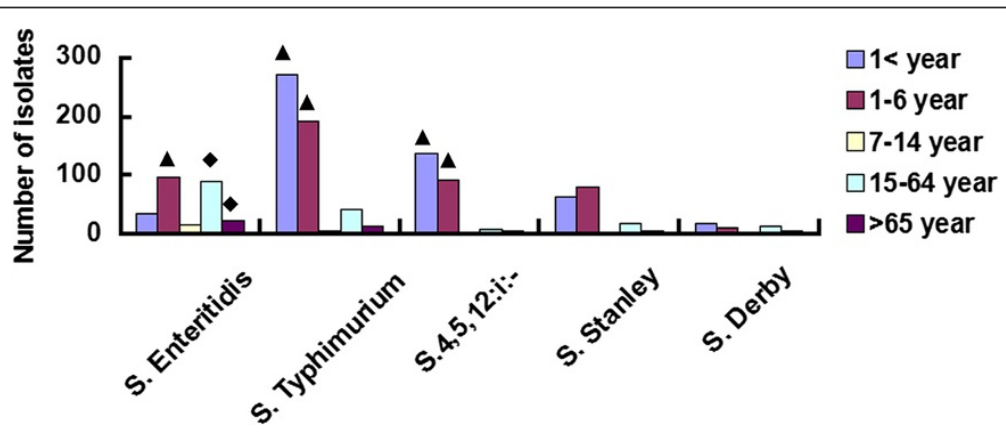

Figure 3 The prevalence of top 5 serovars among the total isolates within different age groups. Less than one years old $(<1)$ : newborn baby group; between 1 and 6 years old (1-6): Infants group; between 7 and 15 years old (7-14): juvenile; between 15 and 64 years old (7-14): adults group; more than 65 years old $(>65)$ : elderly group. The " $\mathbf{\Delta}$ " mark the predominant serovar in less than 6 years old group, and " $\bullet$ "indicate the most prevalent serovar in adults. 
Table 2 Antimicrobial susceptibility among 1764 Salmonella enterica isolates from humans with infections in Guangdong province, China, during 2007 and 2012, and MDR in each of the top 11 serovars

\begin{tabular}{|c|c|c|c|c|c|c|c|c|c|c|c|c|c|c|}
\hline \multirow[b]{2}{*}{$\begin{array}{l}\text { S. enterica } \\
\text { serovar }\end{array}$} & \multirow[b]{2}{*}{$\begin{array}{l}\text { No. of } \\
\text { isolates }\end{array}$} & \multicolumn{12}{|c|}{ No. of isolates resistant to indicated agent at the indicated breakpoint (\% resistance) ${ }^{a}$} & \multirow[b]{2}{*}{ MDR } \\
\hline & & $\begin{array}{c}\text { AMP } \\
\text { Ampicillin }\end{array}$ & $\begin{array}{c}\text { CAZ } \\
\text { Ceftazidime }\end{array}$ & $\begin{array}{c}\text { CTX } \\
\text { Cefotaxime }\end{array}$ & $\begin{array}{c}\text { FEP } \\
\text { Cefepime }\end{array}$ & $\begin{array}{c}\text { NAL } \\
\text { Nalidixic } \\
\text { acid }\end{array}$ & $\begin{array}{c}\text { CIP } \\
\text { Ciprofloxacin }\end{array}$ & $\begin{array}{c}\text { SMX } \\
\text { Sulfamethoxazole }\end{array}$ & $\begin{array}{c}\text { TMP } \\
\text { Trimethoprim }\end{array}$ & $\begin{array}{c}\text { TCY } \\
\text { Tetracycline }\end{array}$ & $\begin{array}{c}\text { GEN } \\
\text { Gentamicin }\end{array}$ & $\begin{array}{c}\text { STR } \\
\text { Streptomycin }\end{array}$ & $\begin{array}{c}\mathrm{CHL} \\
\text { Chloramphenicol }\end{array}$ & \\
\hline Enteritidis & 257 & $143(55.64)$ & 11(4.28) & 14(5.45) & 18(7.01) & $212(82.49)$ & $11(4.28)$ & $141(54.86)$ & 23(89.49) & $76(29.57)$ & 23(8.95) & $110(42.81)$ & 10(3.89) & 116(45.14) \\
\hline Typhimurium & 523 & 453(86.62) & $43(82.21)$ & $68(13.01)$ & 69(13.19) & 437(83.56) & $60(11.47)$ & 455(86.99) & $337(64.44)$ & $457(87.38)$ & $340(65.01)$ & $391(74.76)$ & $381(72.85)$ & $454(86.80)$ \\
\hline 1,4,5,12:::- & 244 & $220(90.16)$ & $18(7.37)$ & $30(12.29)$ & $29(11.88)$ & 182(74.59) & $28(11.47)$ & $220(90.16)$ & $128(52.46)$ & 228(93.44) & $141(57.78)$ & $164(67.21)$ & $164(67.21)$ & 218(89.34) \\
\hline Stanley & 167 & $14(8.38)$ & 10(5.99) & $11(6.58)$ & $9(5.39)$ & 19(11.37) & $8(4.79)$ & $35(20.96)$ & $14(8.38)$ & $20(11.98)$ & $13(7.78)$ & $25(14.97)$ & 13(7.78) & $18(10.78)$ \\
\hline Derby & 46 & 15(26.08) & $2(4.35)$ & $3(6.52)$ & $3(6.52)$ & 17(36.96) & $4(8.69)$ & $35(76.08)$ & $14(30.44)$ & $22(47.83)$ & $14(30.44)$ & 19(41.30) & $16(34.78)$ & $15(32.61)$ \\
\hline Rissen & 36 & $26(72.22)$ & $3(8.33)$ & $5(13.89)$ & $6(16.67)$ & $5(13.89)$ & $1(2.78)$ & $23(63.89)$ & 30(83.33) & $31(86.11)$ & $7(19.44)$ & $16(44.44)$ & $5(13.89)$ & $15(41.67)$ \\
\hline Weltevreden & 35 & $3(8.57)$ & $2(5.71)$ & $2(5.71)$ & $2(5.71)$ & $4(11.43)$ & $1(2.86)$ & $8(22.86)$ & $4(11.43)$ & $6(17.14)$ & $3(8.57)$ & 6(17.14) & $2(5.71)$ & $3(8.57)$ \\
\hline Infantis & 28 & $23(82.14)$ & 0 & $3(10.71)$ & $2(7.14)$ & 23(82.14) & 0 & $23(82.14)$ & 19(67.86) & 19(67.86) & $4(14.28)$ & $4(14.28)$ & $21(75)$ & $21(75)$ \\
\hline Agona & 24 & $4(16.67)$ & 0 & $1(4.17)$ & $3(12.5)$ & $4(16.67)$ & $3(12.5)$ & $7(29.17)$ & $3(12.5)$ & $8(33.33)$ & $4(16.67)$ & $5(20.83)$ & $5(20.83)$ & $6(25)$ \\
\hline Albany & 22 & $21(95.45)$ & $3(13.63)$ & $4(18.18)$ & $4(18.18)$ & 20(90.9) & $1(4.54)$ & $22(100)$ & 20(90.9) & $21(95.45)$ & $4(18.18)$ & 7(31.81) & $21(95.45)$ & 20(90.9) \\
\hline Newport & 19 & $3(15.79)$ & $2(10.53)$ & 1(5.26) & 0 & $4(21.05)$ & $1(5.26)$ & $5(26.31)$ & $3(15.79)$ & $6(31.57)$ & $2(10.53)$ & $5(26.31)$ & $3(15.79)$ & $4(21.05)$ \\
\hline Typhi & 4 & $1(25)$ & 0 & $1(25)$ & 0 & $1(25)$ & 0 & $1(25)$ & $1(25)$ & $1(25)$ & $1(25)$ & $1(25)$ & $1(25)$ & $1(25)$ \\
\hline $\begin{array}{l}\text { Paratyphi } \\
(A+B+C)\end{array}$ & 24 & $5(20.83)$ & $2(8.33)$ & $1(4.16)$ & $1(4.16)$ & $5(20.83)$ & $1(4.16)$ & $4(16.66)$ & 0 & $2(8.33)$ & $1(4.16)$ & $4(16.66)$ & 0 & $3(12.5)$ \\
\hline $\begin{array}{l}\text { Other } \\
114 \text { NTS } \\
\text { serovars }\end{array}$ & 335 & 106(31.64) & 28(8.35) & $32(9.55)$ & $23(6.86)$ & 119(35.52) & $23(6.86)$ & $141(42.09)$ & 106(31.06) & $161(31.64)$ & $54(16.11)$ & 121(36.11) & 72(21.49) & 103(30.74) \\
\hline 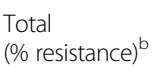 & & 1037(58.78) & $124(7.03)$ & $176(9.98)$ & 169(9.58) & $1052(59.64)$ & $142(8.05)$ & $1120(63.49)$ & 702(39.79) & $1058(59.97)$ & $611(34.64)$ & $878(49.77)$ & $714(40.48)$ & $997(56.52)$ \\
\hline
\end{tabular}

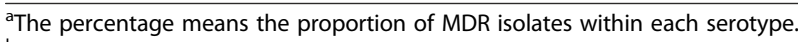

${ }^{\mathrm{b}}$ The percentage means the proportion of MDR isolates in total 1764 isolates. 


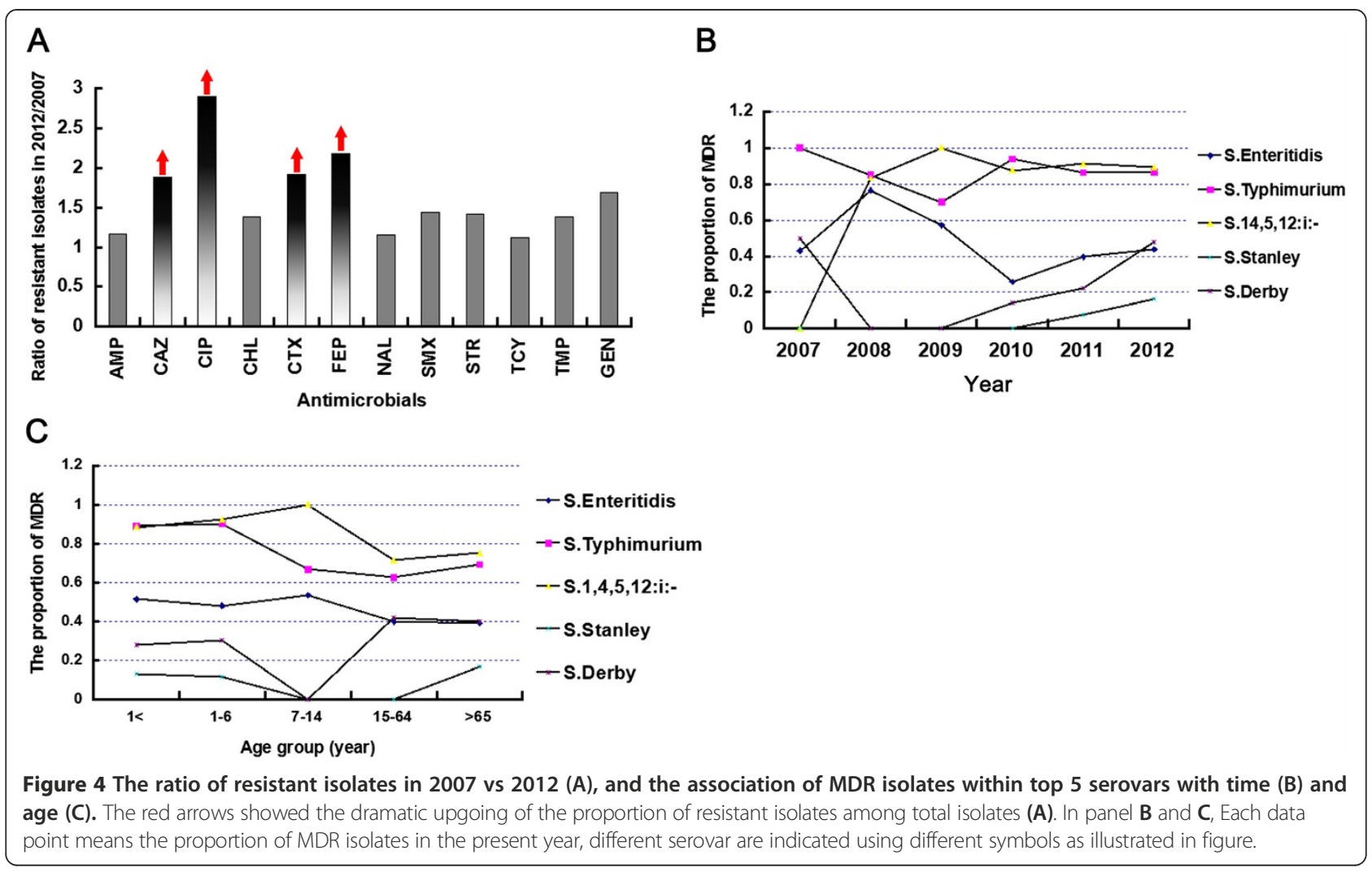

\section{Pulsed field gel electrophoresis}

Among the 1179 isolates of Salmonella analyzed by PFGE, 513 different PFGE profiles were observed (Table 3). Each serovar corresponded to a single clade, while a few isolates could be clustered into other serovar clades (data not show). For the Salmonella Typhimurium and Salmonella 4,5,12:i:- complex clades, 286 PFGE profiles were observed. The PFGE profile, JPXX01.GD0004, was most prevalent, occurring in 21.37\% (150/694) of isolates. For Salmonella Enteritidis, profiles JEGX01.GD0006 ( $\mathrm{n}=50$, 21.55\%) and JEGX01.GD0007 ( $\mathrm{n}=50,21.55 \%)$ were the most common patterns. For Salmonella Stanley, the JNGX01.GD0006 and JNGX01.GD0007 profiles were most common patterns, while no predominant PFGE patterns were observed either in Salmonella Derby (24 PFGE profiles) or other serovars (53 PFGE profiles). The association analysis among the predominant PFGE patterns, MDR, age and geographic location showed that ACSSuT was the predominant MDR pattern in the Salmonella Typhimurium \& S. 4,5,12:i:-complex clade, while ASSuT$\mathrm{Nal}$ (nalidixic acid)and ASSu-Nal were the predominant MDR profiles in Salmonella Enteritidis. No dominant MDR patterns were observed in Salmonella Stanley, Salmonella Derby or other serovars (Table 3). The predominant PFGE patterns of the Salmonella Typhimurium \& S. 4,5,12:i:-complex clade and Salmonella Stanley mainly prevalent in infants ( $<6$ years). However, no obvious relationship was observed between the PFGE profiles and geographic location (Table 3).

\section{Discussion}

The current study indicated the serovar profiles of Salmonella enterica in diarrheal outpatients, and confirmed widespread MDR and reduced ciprofloxacin and thirdgeneration cephalosporin susceptibility among NTS isolates from Guangdong province. Although typhoid Salmonella occurred in a few cases, most of them showed no resistance to ciprofloxacin or third-generation cephalosporins. These results were in agreement with the general knowledge of the epidemiology of salmonellosis [6].

The serovar profile data showed that Salmonella Typhimurium became the dominant serovar in 2008. Salmonella 4,5,12:i:- is a monophasic variant of Salmonella Typhimurium, which is very similar at the molecular level [24,25]. Therefore, the complex group of Salmonella Typhimurium \& 4,5,12:i:- ranked first in endemic serovars of Salmonella in Guangdong. These data differ from those in Europe and Latin America where Salmonella Enteritidis is the most common serovar, followed by Salmonella Typhimurium $[26,27]$. In contrast, the Guangdong data agree with those in Africa and North America where Salmonella Typhimurium seems to be more common than Salmonella Enteritidis [28]. However, we still do not know the exact reasons for this shifting from Salmonella Enteritidis to Salmonella 
Table 3 The association analysis among predominant PFGE patterns in each serovar, MDR, age and geography location

\begin{tabular}{|c|c|c|c|c|c|}
\hline & S. Typhimurium \& S. 4,5,12:i:- & S. Enteritidis & S. Stanley & S. Derby & Others serovars \\
\hline Number of PFGE pattern (n) & $286(n=702)^{a}$ & $78(n=232)$ & $72(n=150)$ & $24(n=28)$ & $53(n=77)$ \\
\hline \multirow[t]{4}{*}{ Predominant PFGE pattern $(n, \%)^{b}$} & JPXX01.GD0004 & JEGX01.GD0006 & JNGX01.GD0006 & None & None \\
\hline & $(n=150,21.37 \%)$ & $(n=50,21.55 \%)$ & $(n=23,15.33 \%)$ & & \\
\hline & & JEGX01.GD0007 & JNGX01.GD0007 & & \\
\hline & & $(n=50,21.55 \%)$ & $(n=29,19.33 \%)$ & & \\
\hline Age $\leq 6$ years & 141 & 51 & 44 & & \\
\hline$>6$ years & 9 & 49 & 8 & & \\
\hline \multirow[t]{12}{*}{ Location } & Dongguan & Dongguan & Dongguan & & \\
\hline & Foshan & Foshan & Foshan & & \\
\hline & Guangzhou & Guangzhou & Guangzhou & & \\
\hline & Jiangmen & Heyuan & Jieyang & & \\
\hline & Jieyang & Huizhou & Maoming & & \\
\hline & Maoming & Jiangmen & Zhanjiang & & \\
\hline & Shantou & Jieyang & Zhaoqing & & \\
\hline & Yangjiang & Maoming & Zhongshan & & \\
\hline & Zhaoqing & Shaoguan & Zhuhai & & \\
\hline & Zhongshan & Yangjiang & & & \\
\hline & Zhuhai & Zhongshan & & & \\
\hline & & Zhuhai & & & \\
\hline \multirow[t]{5}{*}{ MDR pattern $(n, \%)^{c}$} & ACSSuT $(99,66.00 \%)$ & JEGX01.GD0006 & JNGX01.GD0006 & & \\
\hline & & None & None & & \\
\hline & & JEGX01.GD0007 & JNGX01.GD0007 & & \\
\hline & & ASSuT-Nal $(30,30 \%)$ & None & & \\
\hline & & ASSu-Nal $(17,17 \%)$ & & & \\
\hline
\end{tabular}

${ }^{a} \mathrm{n}=$ number of isolates which PFGE data available.

${ }^{b} n=$ number of isolates in the predominant PFGE pattern, $\%=$ percentage in isolates with PFGE data.

$c_{n}=$ number of MDR isolates in the predominant PFGE pattern, $\%=$ percentage in predominant PFGE pattern isolates.

Typhimurium and Salmonella 4,5,12:i:-. As we know, Salmonella Typhimurium can be found in a wide range of animal species and is most commonly observed in pigs, while the most common reservoirs of Salmonella Enteritidis are eggs and chickens [29]. Therefore, it seems likely that the wider reservoir pattern for Salmonella Typhimurium and Salmonella 4,5,12:i:- provides them with more opportunities to come in contact with people and other reservoirs, resulting in the fast expansion of these two serovars. Another possible explanation for the increased detection of Salmonella Typhimurium and Salmonella 4,5,12:i:- in Guangdong is the updated training program for sampling and culture in 2009. After training, the total number of sampling and isolates increased significantly in 2009 (Table 1). Meanwhile, we found that both Salmonella Typhimurium and Salmonella 4,5,12:i:- affected infants, whereas Salmonella Enteritidis affected adults. The different eating habits of infants and adults or special host adaptations may explain the observed distribution pattern. Salmonella Stanley and Derby were also commonly found in Guangdong as observed in other regions of the world $[26,28]$. Several other serovars that are common in many countries were also found in Guangdong, such as Salmonella Rissen, Salmonella Weltevreden, Salmonella Infantis, Salmonella Agona, Salmonella Albany and Salmonella Newport $[26,28]$. Likely, the frequent global travel, communication and trading that occur in Guangdong favor the transmission of these serovars, as similar trends have been verified in Asia [30], Africa and Europe [31].

The antimicrobial susceptibility analyses cover antimicrobial resistance patterns in 15 of 21 cities in Guangdong where the Salmonella situation had been poorly characterized. Indeed, previous data about Salmonellosis in Guangdong comprise few studies [15-18]. Comparing these studies illustrates the emergence of antimicrobial resistance except for the dramatic increase of resistance to ciprofloxacin and third-generation cephalosporins, which we have summarized in Guangdong province for the first time here. A similar increase in resistance to antibiotics was observed in fish and chicken in Guangdong [32]. The 
observed antibiotic resistance trend in Guangdong is very similar to those described in the Guangxi [33] and Henan provinces [14] in China. The major difference is the higher ciprofloxacin resistance observed in Henan than Guangdong (54\% vs 8.05\%). Meanwhile we found resistance to both ciprofloxacin and cephalosporins in Salmonella Typhimurium, Salmonella 4,5,12:i:- and Salmonella Enteritidis. Ciprofloxacin is currently the recommended the first-line antibiotic by the World Health Organization for the treatment of salmonellosis [34]. Third-generation cephalosporins are considered alternative drugs for salmonellosis treatment [34]. Hence, the resistance to fluoroquinolones and third-generation cephalosporins will pose a great challenge for the effective treatment of salmonellosis. Particularly, the dramatic increase of resistance to ciprofloxacin and third-generation cephalosporins in infants is a warning signal for a prudent application of these antibiotics in clinical settings. This situation in Guangdong seems completely different from particular areas in Africa where Salmonella are highly susceptible to antibiotics [35]. Therefore, close surveillance of Salmonella and their microbial resistance patterns is essential to prevent the expansion of MDR clonal populations [36]. Furthermore, the reduced ciprofloxacin susceptibility was, in all cases, associated with nalidixic acid resistance. Therefore, the detection of nalidixic acid resistance remains a valuable test to screen for reduced ciprofloxacin susceptibility in Guangdong.

PFGE typing implicated a relatively high diversity in all serotypes, indicating that the human cases were most likely sporadic; this interpretation agrees with unrelated epidemiological background of all of the cases [37]. We found few dominant PFGE patterns in past and recent isolates of Salmonella Typhimurium, Salmonella 4,5,12:i:-, Salmonella Enteritidis and Salmonella Stanley. Thus, these common patterns seem to have been constant over time. Meanwhile, resistant patterns of each predominant PFGE pattern also differed from each other. Therefore, the different PFGE patterns perhaps came from different clonal lineage. Even so, the obtained PFGE profiles and representative strains are available for comparison with profiles obtained of Salmonella from other countries to track the asymptomatic carriers and identify the circulation of major clones worldwide. However, as we did not determine the genotype (such as MLST or MLVA) of the dominant serovars, such as in the Salmonella Typhimurium and Salmonella 4,5,12:i:- isolates, we do not know if these serovars belong to the same lineages that are found in other continents; therefore, it would be of crucial importance to determine the genotype to link the possible transmission pathway of each predominant serovar in future studies [38].

\section{Conclusion}

In conclusion, Salmonella Typhimurium and Salmonella 4,5,12:i:- were the most common serovars isolated during the observation period from 2007 to 2012 in Guangdong, mainly causing salmonellosis in infants, whereas Salmonella Enteritidis mainly caused salmonellosis in adults. In 2008, a serovar shift from Salmonella Enteritidis to Salmonella Typhimurium became apparent. A high burden of multidrug resistant strains and an increasing incidence of quinolone and third-generation cephalosporin resistance were observed. Close surveillance of Salmonella and their microbial resistance patterns, using combined molecular tools, such as PFGE, multilocus sequence typing (MLST) and multiple locus VNTR analysis (MLVA), is therefore dedicate to prevent outbreaks of disease, track the potential transmission pathways and rationalize the antimicrobial therapy of salmonellosis.

\section{Additional file}

Additional file 1: Table S1. The isolates collected in this study, patient information, antimicrobial susceptibility test profiles and PFGE results. The isolates resistant to all tested antimicrobials are labeled with red.

\section{Competing interests}

The authors declare that they have no financial or non-financial competing interests.

\section{Authors' contributions}

$\mathrm{BK}, \mathrm{DH}, \mathrm{XL}$ and $\mathrm{ZL}$ contributed to acquisition of data. JS and BK conducted the analysis, interpretation of data and manuscript drafting. CK contributed to the conception and design of this study and the manuscript revision. All authors read and approved the final manuscript.

\section{Acknowledgments}

This project was supported by the China-U.S. Collaborative Program on Emerging and Re-Emerging Infectious Diseases and the Medical Scientific Research Foundation in Guangdong (1U2GGH000018-01), and Science and Technology Planning Project of Guangdong Province Program

(2012B060400012). The authors also acknowledge support from the WHO Global Foodborne Infections Network.

\section{Author details}

${ }^{1}$ Institute of Microbiology, Guangdong Provincial Center for Disease Control and Prevention, 511430 Guangzhou, China. ${ }^{2}$ Guangdong Provincial Institute of Public Health, Guangdong Provincial Center for Disease Control and Prevention, 511430 Guangzhou, China.

Received: 12 February 2014 Accepted: 9 June 2014

Published: 17 June 2014

\section{References}

1. Switt Al, Soyer Y, Warnick LD, Wiedmann M: Emergence, distribution, and molecular and phenotypic characteristics of Salmonella enterica serotype 4,5,12:i. Foodborne Pathog Dis 2009, 6(4):407-415.

2. Crump JA, Luby SP, Mintz ED: The global burden of typhoid fever. Bull World Health Organ 2004, 82(5):346-353.

3. Ochiai RL, Acosta CJ, Danovaro-Holliday MC, Baiqing D, Bhattacharya SK, Agtini MD, Bhutta ZA, Canh do G, Ali M, Shin S, Wain J, Page AL, Albert MJ, Farrar J, Abu-Elyazeed R, Pang T, Galindo CM, von Seidlein L, Clemens JD, Domi Typhoid Study Group: A study of typhoid fever in five Asian countries: disease burden and implications for controls. Bull World Health Organ 2008, 86(4):260-268.

4. Crump JA, Mintz ED: Global trends in typhoid and paratyphoid Fever. Clin Infect Dis 2010, 50(2):241-246.

5. Parry CM, Hien T,, Dougan G, White NJ, Farrar JJ: Typhoid fever. N Engl J Med 2002, 347(22):1770-1782. 
6. Majowicz SE, Musto J, Scallan E, Angulo FJ, Kirk M, O'Brien SJ, Jones TF, Fazil A Hoekstra RM, International Collaboration on Enteric Disease 'Burden of Illness' Studies: The global burden of nontyphoidal Salmonella gastroenteritis. Clin Infect Dis 2010, 50(6):882-889.

7. Reddy EA, Shaw AV, Crump JA: Community-acquired bloodstream infections in Africa: a systematic review and meta-analysis. Lancet Infect Dis 2010, 10(6):417-432.

8. Varma JK, Molbak K, Barrett TJ, Beebe JL, Jones TF, Rabatsky-Ehr T, Smith KE, Vugia DJ, Chang HG, Angulo FJ: Antimicrobial-resistant nontyphoidal Salmonella is associated with excess bloodstream infections and hospitalizations. J Infect Dis 2005, 191(4):554-561.

9. Okeke IN, Aboderin OA, Byarugaba DK, Ojo KK, Opintan JA: Growing problem of multidrug-resistant enteric pathogens in Africa. Emerg Infect Dis 2007, 13(11):1640-1646.

10. Cui S, Li J, Sun Z, Hu C, Jin S, Guo Y, Ran L, Ma Y: Ciprofloxacin-resistant Salmonella enterica serotype Typhimurium, China. Emerg Infect Dis 2008, 14(3):493-495

11. Ling JM, Chan EW, Cheng AF: Molecular epidemiological analysis of Salmonella enterica serotype Derby infections in Hong Kong. J Infect 2001, 42(2):145-153.

12. Ling JM, Koo IC, Kam KM, Cheng AF: Antimicrobial susceptibilities and molecular epidemiology of Salmonella enterica serotype enteritidis strains isolated in Hong Kong from 1986 to 1996. J Clin Microbiol 1998, 36(6):1693-1699.

13. Lo NW, Chu MT, Ling JM: Increasing quinolone resistance and multidrug resistant isolates among Salmonella enterica in Hong Kong. J Infect 2012, 65(6):528-540

14. Xia S, Hendriksen RS, Xie Z, Huang L, Zhang J, Guo W, Xu B, Ran L, Aarestrup FM: Molecular characterization and antimicrobial susceptibility of Salmonella isolates from infections in humans in Henan Province. Chin J Clin Microbiol 2009, 47(2):401-409.

15. Deng X, Ran L, Wu S, Ke B, He D, Yang X, Zhang Y, Ke C, Klena JD, Yan M, Feng Z, Kan B, Liu X, Mikoleit M, Varma JK: Laboratory-based surveillance of non-typhoidal Salmonella infections in Guangdong Province. China. Foodborne Pathog Dis 2012, 9(4):305-312.

16. He DM, Ke BX, Deng XL, Ke CW, Liang ZM, Tan HL, Li BS, Liu MZ, Chen JD: [Surveillance and analysis on the pathogenic features of Salmonella in Guangdong province in 2010]. Zhonghua Yu Fang Yi Xue Za Zhi 2010, 46(5):424-429

17. Ke BX, Deng XL, Zhang LH, Chen JL, Ke CW, Guo C, Cao HY, Lin W, Tan HL, Li BS: Surveillance and pathogenic analysis on non-typhoidal Salmonella in Guangdong province, 2007. Zhonghua Liu Xing Bing Xue Za Zhi 2008, 29(12):1199-1203.

18. Ke BX, Deng XL, Li BS, Tan HL, He DM, Liu MZ, Chen JD, Ke CW: [Surveillance on Salmonella infection in Guangdong province, 2008-2009]. Zhonghua Liu Xing Bing Xue Za Zhi 2011, 32(8):789-792.

19. Martin $\sqcup$, Fyfe M, Dore K, Buxton JA, Pollari F, Henry B, Middleton D, Ahmed R, Jamieson F, Ciebin B, McEwen SA, Wilson JB, Multi-Provincial Case-Control Study Steering Committee: Increased burden of illness associated with antimicrobial-resistant Salmonella enterica serotype Typhimurium infections. J Infect Dis 2004, 189(3):377-384.

20. Organization WHO: The Global Foodborne Infections Network (GFN): Strategic Plan 2011-2015. World Health Organization; 2010 (www.who.int/gfn/ publications/strategic_plan_2011/en/)

21. Patrick G, François W: Antigenic formulae of the Salmonella Serovars. Paris: World Health Organization, Institut Pasteur; 2007:166.

22. CLSI: Performance standards for antimicrobial susceptibility testing; Twentysecond informational supplement Wayne, PA: Clinical and Laboratory Standards Institute; 2012:188.

23. Ribot EM, Fair MA, Gautom R, Cameron DN, Hunter SB, Swaminathan B, Barrett TJ: Standardization of pulsed-field gel electrophoresis protocols for the subtyping of Escherichia coli 0157:H7, Salmonella, and Shigella for PulseNet. Foodborne Pathog Dis 2006, 3(1):59-67.

24. Zamperini K, Soni V, Waltman D, Sanchez S, Theriault EC, Bray J, Maurer JJ: Molecular characterization reveals Salmonella enterica serovar 4,[5],12:i:- from poultry is a variant Typhimurium serovar. Avian Dis 2007, 51(4):958-964.

25. Dionisi AM, Graziani C, Lucarelli C, Filetici E, Villa L, Owczarek S, Caprioli A, Luzzi I: Molecular characterization of multidrug-resistant strains of Salmonella enterica serotype Typhimurium and Monophasic variant (S. 4,[5],12:i:-) isolated from human infections in Italy. Foodborne Pathog Dis 2009, 6(6):711-717.
26. Galanis E, Lo Fo Wong DM, Patrick ME, Binsztein N, Cieslik A, Chalermchikit T, Aidara-Kane A, Ellis A, Angulo FJ, Wegener HC: Web-based surveillance and global Salmonella distribution, 2000-2002. Emerg Infect Dis 2006, 12(3):381-388.

27. Olsen SJ, Bishop R, Brenner FW, Roels TH, Bean N, Tauxe RV, Slutsker L: The changing epidemiology of Salmonella: trends in serotypes isolated from humans in the United States, 1987-1997. J Infect Dis 2001, 183(5):753-761.

28. Herikstad H, Motarjemi Y, Tauxe RV: Salmonella surveillance: a global survey of public health serotyping. Epidemiol Infect 2002, 129(1):1-8.

29. Yang B, Xi M, Wang X, Cui S, Yue T, Hao H, Wang Y, Cui Y, Alali WQ, Meng J, Walls I, Wong DM, Doyle MP: Prevalence of Salmonella on raw poultry at retail markets in China. J Food Prot 2011, 74:1724-1728.

30. Hakanen A, Kotilainen P, Huovinen P, Helenius H, Siitonen A: Reduced fluoroquinolone susceptibility in Salmonella enterica serotypes in travelers returning from Southeast Asia. Emerg Infect Dis 2001, 7(6):996-1003.

31. Lunguya O, Lejon V, Phoba MF, Bertrand S, Vanhoof R, Glupczynski Y, Verhaegen J, Muyembe-Tamfum JJ, Jacobs J: Antimicrobial resistance in invasive non-typhoid Salmonella from the Democratic Republic of the Congo: emergence of decreased fluoroquinolone susceptibility and extended-spectrum beta lactamases. PLoS Negl Trop Dis 2013, 7(3):e2103.

32. Broughton El, Walker DG: Prevalence of antibiotic-resistant Salmonella in fish in Guangdong. Chin Foodborne Pathog Dis 2009, 6(4):519-521.

33. Jin Y: Enteric fever in south China: Guangxi province. J Infect Dev Ctries 2008, 2(4):283-288.

34. Orgnization WHO: WHO recommendations on the management of diarrhoed and pneumonia in HIV-infected infants and children. WHO Library 2010, WHO reference number: 9789241548083 . (www.who.int/maternal child_adolescent/ documents/9789241548083/en/).

35. Saba CK, Escudero JA, Herrera-Leon S, Porrero MC, Suarez M, Dominguez L, Demuyakor B, Gonzalez-Zorn B: First identification of Salmonella Urbana and Salmonella Ouakam in humans in Africa. J Infect Dev Ctries 2013, 7(10):691-695

36. Wong MH, Yan M, Chan EW, Liu LZ, Kan B, Chen S: Expansion of Salmonella enterica serovar Typhimurium ST34 clone carrying multiple resistance determinants in China. Antimicrob Agents Chemother 2013, 57(9):4599-4601.

37. Proctor ME, Brosch R, Mellen JW, Garrett LA, Kaspar CW, Luchansky JB: Use of pulsed-field gel electrophoresis to link sporadic cases of invasive listeriosis with recalled chocolate milk. Appl Environ Microbiol 1995, 61(8):3177-3179

38. Feasey NA, Dougan G, Kingsley RA, Heyderman RS, Gordon MA: Invasive non-typhoidal Salmonella disease: an emerging and neglected tropical disease in Africa. Lancet 2012, 379(9835):2489-2499.

doi:10.1186/1471-2334-14-338

Cite this article as: Ke et al:: Serovar distribution, antimicrobial resistance profiles, and PFGE typing of Salmonella enterica strains isolated from 2007-2012 in Guangdong, China. BMC Infectious Diseases 2014 14:338

\section{Submit your next manuscript to BioMed Central and take full advantage of:}

- Convenient online submission

- Thorough peer review

- No space constraints or color figure charges

- Immediate publication on acceptance

- Inclusion in PubMed, CAS, Scopus and Google Scholar

- Research which is freely available for redistribution 